\title{
Atypical Variant of Guillain Barre Syndrome in a Patient with COVID-19
}

\author{
Megan M. Lowery ${ }^{1 *}$, Muhammad Taimur Malik², Joseph Seemiller², Cynthia S. Tsai1 \\ 1 Pulmonary/Critical Care Medicine, Geisinger Medical Center, Danville PA, USA \\ 2 Geisinger Neuroscience Institute, Geisinger Medical Center, Danville, PA, USA
}

\begin{abstract}
Objective: A rare variant Miller Fisher Syndrome overlap with Guillain Barre Syndrome is described in an adult patient with SARS-COV-2 infection. Case Presentation: The clinical course of a 45-year-old immunosuppressed man is summarized as a patient who developed ataxia, ophthalmoplegia, and areflexia after upper respiratory infection symptoms began. A nasopharyngeal swab was positive for COVID-19 polymerase chain reaction. He progressed to acute hypoxemic and hypercapnic respiratory failure requiring intubation and rapidly developed tetraparesis. Magnetic resonance imaging of the spine was consistent with Guillain Barre Syndrome. However, the clinical symptoms, along with positive anti-GQ1B antibodies, were consistent with Miller Fisher Syndrome and Guillain Barre Syndrome overlap. The patient required tracheostomy and had limited improvement in his significant neurological symptoms after several months. Conclusions: The case demonstrates the severe neurological implications, prolonged recovery and implications in the concomitant respiratory failure of COVID-19 patients with neurological symptoms on the spectrum of disorders of Guillain Barre Syndrome.
\end{abstract}

Keywords: hypoxemic and hypercapnic respiratory failure, COVID-19, SARS-CoV-2, Guillain Barre Syndrome, Miller Fisher Syndrome

Received: 29 June 2020 / Accepted: 20 October 2020

\section{INTRODUCTION}

Severe Acute Respiratory Syndrome Coronavirus-2 (SARS-CoV-2), causing coronavirus disease 2019 (COVID-19) infection, has spread globally, with an increasing number of reported cases of critically ill patients with neurological complications [1]. Neurologic complications of COVID-19 range from headache and anosmia to encephalopathy, seizure, strokes and Guillain-Barre syndrome [1-3]. Guillain Barre Syndrome refers to a group of immunological disorders often characterized by acute or subacute weakness in limbs or cranial nerve innervated muscles. Multiple distinct variations of clinical presentation and pathophysiology help categorize Guillain Barre Syndrome subsets $[4,5]$.

The inciting event that triggers this immunological reaction includes vaccinations, pregnancy, and surgical procedures but is often precipitated by infection $[6,7]$. Common antecedent infections include Campylobacter jejuni, Mycoplasma pneumoniae, Cytomegalovirus, Epstein-Barr virus, influenza A and the Zika virus $[4,8]$. Several coronaviruses, including those that cause Middle East respiratory syndrome (MERS), severe acute respiratory syndrome (SARS) and COVID-19 have also been implicated $[3,9,10]$.

The case is presented of a COVID-19 infected patient initially presenting to the hospital with symptoms of Miller Fisher Syndrome, a variant of Guillain Barre Syndrome, characterized by areflexia, ataxia and ophthalmoplegia. The patient later developed diffuse weakness, more typically seen in other Guillain Barre Syndrome variants as well as in overlapping Miller Fisher Syndrome/Guillain Barre Syndrome.

\section{Case Presentation}

A 45-year-old gentleman presented at the Geisinger Bloomsburg Hospital, Bloomsburg, PA, USA. He had self-referred to the hospital emergency department (ED) after developing gait ataxia and weakness at home. On admission, it was documented that he had complained for the past two weeks of sinus congestion, cough with sputum production, chest tightness, and dyspnoea. His past medical history was noteworthy for dyslipidemia, hypertension and Crohn's disease, the latter being treated with adalimumab. It was noted that 
he had left facial and bilateral lower extremity numbness and dysgeusia. Tele-stroke examination showed he had an NIH Stroke Scale of 2. Computerized tomography angiography of the head and neck and chest radiography, completed upon presentation at the ED, showed no acute abnormalities.

The patient was admitted to Geisinger Bloomsburg Hospital. Nasopharyngeal COVID-19 polymerase chain reaction testing was performed in the ED at 13:26 hours. The results were positive.

On Day 1, post-admission, 200 milligrams hydroxychloroquine sulphate (Appco Pharmaceuticals, Piscataway, NJ), twice daily was initiated and given parenterally. He started a five-day course according to the institution's COVID-19 protocol, Day 1 post-admission.

The patient's neurologic examination and weakness worsened on Day 2 post-admission, progressing to dysphagia and quadriparesis. He developed acute hypoxemic with hypercapnic respiratory failure, which required intubation and mechanical ventilation on Day 2 post-admission at 0211 hours. A post-intubation CTchest scan undertaken on Day 2 post-admission, demonstrated new bilateral lower lobe consolidations with air bronchograms (Figure 1).

His neurologic examination revealed bilateral ptosis, cranial nerve 3, 4, and 6 weakness, 2/5 right upper and bilateral lower extremity strength, but $0 / 5$ left upper extremity strength. There was minimal shoulder shrug weakness and global areflexia. Due to his ventilator requirements and disease progression, in the setting of
COVID-19, he was transferred to the Adult Intensive Care Unit (AICU) at Geisinger Medical Centre in Danville, PA, USA, a tertiary care facility on Day 2 postadmission, at 06:00 hours.

The patient underwent a lumbar puncture on Day 2 post-admission to the AICU at 17:51 hours. Cerebrospinal fluid (CSF) demonstrated normal protein and glucose levels with only one white blood cell and four red blood cells present. This result suggested that bacterial meningitis was unlikely. CSF cultures were not obtained due to the limited CSF volume available for analysis.

As the clinical picture resembled GBS, 0.4 grams/ kilogram intravenous immunoglobulin (CSL Behring, Kanakakee, IL) was administered intravenously and prescribed daily for a total of five days starting on Day 2 post-admission to AICU. However, this was stopped on Day 3 post-admission, as there was a concern due to the worsening of his neurological status.

Magnetic Resonance Imaging of the brain and cervical spine on Day 5 post-admission to AICU, showed no acute findings and thus 0.4 grams/kilogram intravenous immunoglobulin (CSL Behring, Kanakakee, IL) was resumed on Day 6 through to Day 8 post-admission to AICU.

CSF analysis, following a second lumbar puncture on Day 8 post-admission to AICU, showed normal protein and glucose levels and negative bacterial, viral and fungal cultures. Due to testing restrictions, a COVID-19 polymerase chain reaction test on the CSF could
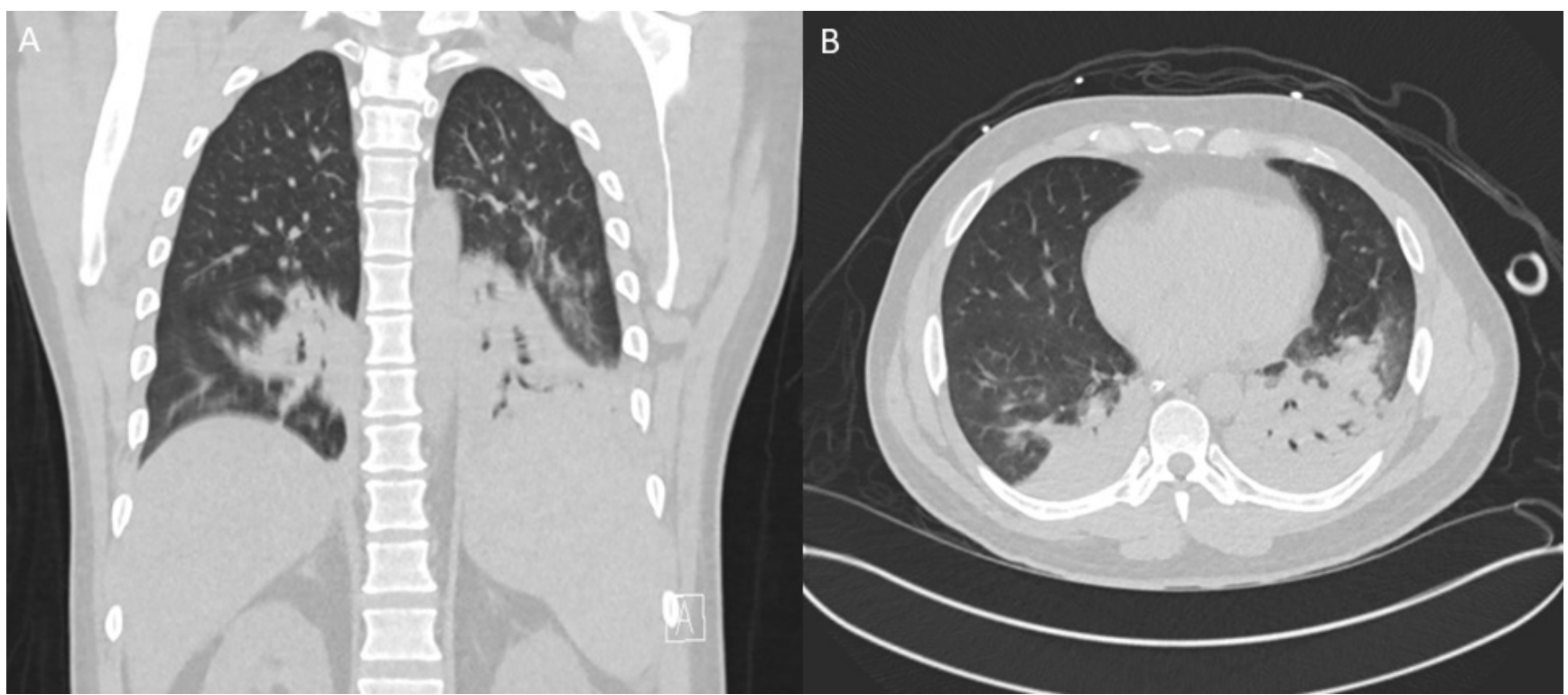

Fig. 1. (A) Coronal and (B) Axial section of the CT chest showing bilateral consolidations with air bronchograms worse on the left than the right lung with left greater than right lower lobe atelectasis 
not be obtained. Tracheal aspirate culture grew betalactamase resistant Haemophilus influenza. The patient received 3 grams ampicillin-sulbactam (Meitheal Pharmaceuticals, Chicago, IL) intravenously every 6 hours for six days, from Day 5 to Day 10 post-admission to AICU.

A repeat magnetic resonance imaging scan of the brain, cervical, thoracic, and lumbar spine, taken on Day 14 post-admission to AICU, revealed intrathecal cauda-equina enhancement consistent with Guillain Barre Syndrome (Figure 2). The patient was also positive for anti-GQ1B antibodies, typically seen in the Miller Fisher Syndrome variant of Guillain Barre. Given the patient's neurological examination, imaging and antibody findings, his presentation was consistent with Miller Fisher Syndrome with Guillain Barre Syndrome overlap.

Following intubation on Day 2 post-admission to AICU, the patient was managed on adaptive pressure ventilation-controlled mechanical ventilation (APV) with a tidal volume of $440 \mathrm{~mL}$, set respiratory rate from 14 to 18 breaths per minute, a peak end-expiratory pressure (PEEP) of 8-12 $\mathrm{cmH}_{2} \mathrm{O}$ and fraction of inspired oxygen ( $\mathrm{FiO} 2)$ of $40 \%$. Plateau pressures ranged from $18-27 \mathrm{cmH}_{2} \mathrm{O}$, and static compliance ranged from 35.3-74.6 ml/ $\mathrm{cmH}_{2} \mathrm{O}$. He was intermittently trialled on adaptive support ventilation (ASV) with $100 \%$ minute ventilation, PEEP of $5 \mathrm{cmH}_{2} \mathrm{O}$ and $\mathrm{FiO} 2$ of $40 \%$ in attempts to wean him off ventilator support. As weaning trials were unsuccessful, the ventilator settings were changed to pressure controlled-mechanical ventilation (P-CMV) with a PEEP of $8 \mathrm{cmH}_{2} \mathrm{O}$ and pressure support of $14 \mathrm{cmH}_{2} \mathrm{O}$ with $40 \% \mathrm{FiO} 2$ on day 12 postadmission to AICU. Plateau pressures and static compliance remained the same.

On Day 26 post-admission to AICU, the patient required tracheostomy for his ventilator-dependent respiratory failure. He was able to be weaned to $90 \% \mathrm{~min}$ ute ventilation with a PEEP of $7 \mathrm{cmH}_{2} \mathrm{O}$ on ASV. Due to desaturation events during tracheostomy changes, he was transitioned on Day 33 post-admission to AICU to $\mathrm{P}-\mathrm{CMV}$ with a $\mathrm{FiO} 2$ of $60 \%$, pressure support of $22 \mathrm{~cm}$ $\mathrm{H}_{2} \mathrm{O}$, and a PEEP of $10 \mathrm{cmH}_{2} \mathrm{O}$. He was then weaned down on pressure support and changed to APV with a PEEP of $10 \mathrm{cmH}_{2} \mathrm{O}$ and $40 \% \mathrm{FiO} 2$ on Day 35 postadmission to AICU.

After a prolonged hospitalization of five weeks, the patient was transferred to a long-term acute care hospital for ventilator weaning and physical therapy. The patient spent approximately four inpatient months at the longterm acute care hospital, slowly being weaned off full ventilator support. At the time of writing this report, and five and a half months after the initial diagnoses, he was tolerating a few hours per day on pressure-support ventilation with a PEEP of $5 \mathrm{cmH}_{2} 0$ and $\mathrm{FiO} 2$ of $25 \%$. Otherwise, he was being supported by pressure regulated volume control set with a TV of $480 \mathrm{~mL}$, a PEEP of $5 \mathrm{cmH}_{2} \mathrm{O}$ and $\mathrm{FiO} 2$ of $25 \%$. The patient was able to control his head, right thumb, left foot, right toe, and had extraocular and tongue movements. He could perform shoulder shrugs and communicate via a letter board.
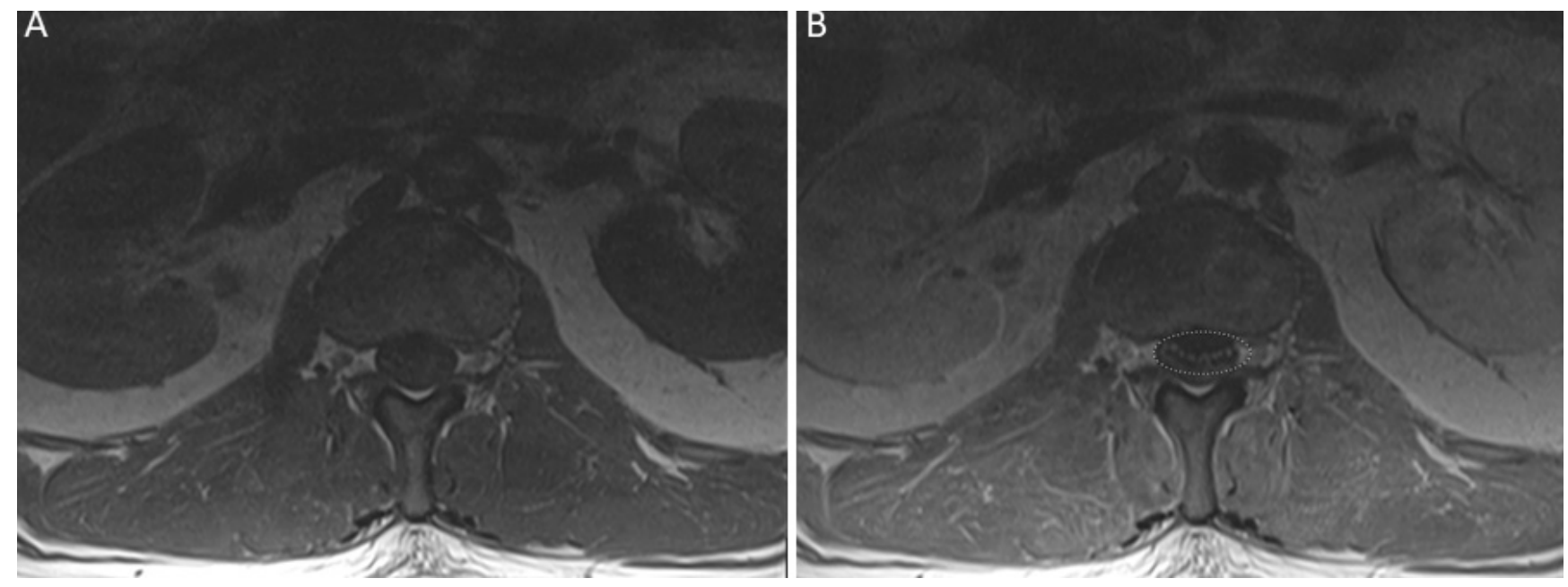

Fig. 2. (A) T 1 Axial sections of the MRI of the lumbar spine without contrast and (B) with contrast showing the ventral nerve root enhancement consistent with a diagnosis of GBS. 


\section{DISCUSSION}

Guillain Barre Syndrome is a generalized term for a heterogeneous group of polyradiculopathies associated with autoimmune responses to stressors with multiple different acute to subacute presentations. Acute inflammatory demyelinating polyradiculopathy (AIDP) and the axonal variants, acute axonal motor neuropathy and acute motor-sensory axonal polyneuropathy, are classically associated with varying degrees of progressive symmetric weakness, sensory abnormalities and hypo- or areflexia. AIDP is a common disorder in North America and is often most easily associated as part of the Guillain Barre Syndrome family of disorders [4]. Miller Fisher Syndrome, Bickerstaff brainstem encephalitis (BBE) and several other less common forms have been described $[4,5,11]$. The Miller Fisher Syndrome variant classically refers to ataxia, areflexia, and ophthalmoplegia but may only portray a part of this triad. Clinical descriptions, antibody features, cerebral spinal fluid analysis and nerve conduction studies can be similar between different Guillain Barre Syndrome variants and at times suggest a spectrum of disease with areas of overlap in presentation as well as pathogenesis.

Limb weakness is more prominent in the demyelinating and axonal variants of the Guillain Barre Syndrome (acute inflammatory demyelinating polyneuropathy (AIDP); acute motor axonal neuropathy (AMAN); acute motor and sensory axonal neuropathy (ASMAN), though limb weakness has been described in Miller Fisher Syndrome [12,13]. Miller Fisher Syndrome is often associated with the antiganglioside antibody anti-GQ1b and cytoalbuminologic dissociation of cerebral spinal fluid, with elevated protein levels in the absence of pleocytosis. However, normal protein levels may be expected in the early course of the disease, as was the case in the patient described [4,11-13]. The anti-GQ1b IgG antibody is also found in BBE and less frequently in other Guillain Barre Syndrome subtypes who had ophthalmoplegia, supporting a common pathogenesis of these disorders sometimes referred to as anti-GQ1b antibody syndrome [14].

There have been multiple accounts in the literature describing overlapping Miller Fisher Syndrome and Guillain Barre Syndrome, where patients with the Miller Fisher Syndrome developed tetraparesis [1517]. Nerve conduction testing in patients with Miller Fisher Syndrome and with overlapping Miller Fisher Syndrome and Guillain Barre Syndrome, have shown reduced sensory nerve action potentials but improved compound motor action potentials on serial nerve conduction testing. These results appear to suggest a reversible motor and sensory conduction delay without demyelination $[15,16,18]$. Another evaluation of nerve conduction testing in a small group of patients with overlapping Miller Fisher Syndrome and Guillain Barre Syndrome showed that two patients had normal sensory nerve conduction, but abnormal motor conduction as evidenced by their acute motor axonal neuropathy. While these patients showed evidence supporting Miller Fisher Syndrome with AMAN, there was no evidence of demyelination on nerve conduction testing [17]. This finding further suggests an axonal motor or sensory component to the overlapping Miller Fisher Syndrome / Guillain Barre Syndrome, rather than a true demyelinating process.

Guillain Barre Syndrome variants have previously been described with other novel coronavirus infections such as SARS and MERS $[9,10,19]$. Neurologic complications of novel coronaviruses may go unrecognized and be underdiagnosed in critically ill patients receiving sedatives and paralytics. Recent cases of Guillain Barre Syndrome and Miller Fisher Syndrome associated with COVID-19 have been described in China and Europe. Gutiérrez-Ortiz et al. (2020) reported a patient with the Miller Fisher Syndrome triad, cytoalbuminologic dissociation for COVID-19, and positive GD1b-IgG antibodies and another patient with areflexia and ophthalmoplegia, the latter being possibly a case of Miller Fisher Syndrome/Guillain Barre Syndrome overlap. The patients' cerebrospinal fluid was negative for COVID-19 [2]. Toscano et al. (2020) published a case series of five patients with Guillain Barre Syndrome-related muscle weakness that was severe and prolonged [3]. Two patients remained mechanically ventilated in the intensive care unit, and two other patients had minimal upper extremity movement at four weeks. Of the five patients, three patients were negative for antiganglioside antibodies, and three had magnetic resonance imaging scans and findings consistent with Guillain Barre Syndrome [3].

The present case demonstrates clinical features of ophthalmoplegia, ataxia and areflexia along with antiGQ1b antibody presence consistent with Miller Fisher Syndrome. However, the patient quickly developed tetraparesis and respiratory failure requiring mechanical ventilation, suggesting that our patient had an overlapping Miller Fisher Syndrome/ Guillain Barre Syndrome. He had onset of neurologic symptoms after 
developing an upper respiratory infection, similarly to other reported cases of the Guillain Barre Syndrome spectrum of disorders but had limited improvement after several weeks $[2,3]$.

The severity of neurologic impairment, with little improvement, is not surprising in patients with overlap syndromes. Funakoshi et al. (2009) reported that 45 patients with overlapping Miller Fisher Syndrome / Guillain Barre Syndrome who required intubation versus those who did not require intubation, presented with cerebellar ataxic gait ( $55 \%$ vs $18 \%, \mathrm{p}=0.04)$. Our patient similarly presented with an ataxic gait. Additionally, Miller Fisher Syndrome / Guillain Barre Syndrome overlap patients required more mechanical ventilation than Guillain Barre Syndrome patients. They also required mechanical ventilation sooner, further suggesting that the clinical course of overlapping Miller Fisher Syndrome/Guillain Barre Syndrome maybe even more severe than patients presenting with Guillain Barre Syndrome alone [17]. A literature search suggested that the present case is the first COVID-19/SARS-CoV-2 case reported in the United States with the Miller Fisher Syndrome triad with anti-GQ1b antibody positivity and tetraparesis with magnetic resonance imaging consistent with Miller Fisher Syndrome/Guillain Barre overlap syndrome.

Limitations of the current report include the lack of cerebrospinal fluid and polymerase chain reaction testing for COVID-19 and the inability to obtain electromyography and nerve conduction studies due to airborne isolation of the patient and limited availability of personal protective equipment.

The neuroinvasive potential of SARS-CoV-2 has been proposed to be due to a novel mechanism specific to the virus [20]. In the current case, the neurological involvement of SARS-CoV2 precipitated severe respiratory failure. The case demonstrates the severity of neurological symptoms and prolonged course of afflicted patients with overlapping Miller Fisher Syndrome / Guillain Barre Syndrome. It suggests that patients presenting to the hospital with neurological symptoms or weakness that could resemble a stroke or Guillain Barre Syndrome should have COVID-19 testing considered as part of their evaluation.

\section{- CONFLICT OF INTEREST}

None to declare.

\section{REFERENCES}

1. Mao L, Jin $H$, Wang $M$, et al. Neurologic Manifestations of Hospitalized Patients with Coronavirus Disease 2019 in Wuhan, China. JAMA Neurol. 2020;77(6):1-9. doi:10.1001/ jamaneurol.2020.1127 [Epub ahead of print]

2. Gutiérrez-Ortiz C, Méndez A, Rodrigo-Rey S, et al. Miller Fisher Syndrome and polyneuritis cranialis in COVID-19 [published online ahead of print, 2020 Apr 17]. Neurology. 2020;10.1212/WNL.0000000000009619. doi:10.1212/ WNL.0000000000009619 [Epub ahead of print]

3. Toscano G, Palmerini F, Ravaglia S, et al. Guillain-Barré Syndrome Associated with SARS-CoV-2. N Engl J Med. 2020;382(26):25742576.

4. Esposito S, Longo MR. Guillain-Barré syndrome. Autoimmun Rev. 2017;16(1):96-101.

5. Wakerley BR, Uncini A, Yuki N, GBS Classification Group. Guillain-Barré and Miller Fisher syndromes--new diagnostic classification. Nat Rev Neurol. 2014;10(9):537-544.

6. Ropper AH. The Guillain-Barré syndrome. N Engl J Med. 1992;326(17):1130-1136.

7. Stoian A, Motataianu A, Bajko Z, Balasa A. Guillain-Barré and Acute Transverse Myelitis Overlap Syndrome Following Obstetric Surgery. J Crit Care Med. 2020;6(1):74-79.

8. Willison HJ, Jacobs BC, van Doorn PA. Guillain-Barré syndrome. Lancet. 2016;388(10045):717-727.

9. Scheidl E, Canseco DD, Hadji-Naumov A, Bereznai B. GuillainBarré syndrome during SARS-CoV-2 pandemic: A case report and review of recent literature. J Peripher Nerv Syst. 2020;25(2):204-207.

10. Wu Y, Xu X, Chen Z, et al. Nervous system involvement after infection with COVID-19 and other coronaviruses. Brain Behav Immun. 2020;87:18-22.

11. Sejvar JJ, Kohl KS, Gidudu J, et al. Guillain-Barré syndrome and Fisher syndrome: case definitions and guidelines for collection, analysis, and presentation of immunization safety data. Vaccine. 2011;29(3):599-612.

12. Snyder LA, Rismondo V, Miller NR. The Fisher variant of GuillainBarré syndrome (Fisher syndrome). J Neuroophthalmol. 2009;29(4):312-324.

13. Mori M, Kuwabara S, Fukutake T, Yuki N, Hattori T. Clinical features and prognosis of Miller Fisher syndrome. Neurology. 2001;56(8):1104-1106.

14. Yoshikawa K, Kuwahara M, Morikawa M, et al. Varied antibody reactivities and clinical relevance in anti-GQ1b antibodyrelated diseases. Neurol Neuroimmunol Neuroinflamm. 2018;5(6):e501.

15. Rajabally YA, Hassan-Smith G, Notturno F, et al. Motor and sensory conduction failure in overlap of Guillain-Barré and Miller Fisher syndrome: two simultaneous cases. J Neurol Sci. 2011;303(1-2):35-38. 
236 - The Journal of Critical Care Medicine 2020;6(4)

16. Umapathi T, Tan EY, Kokubun N, Verma K, Yuki N. Nondemyelinating, reversible conduction failure in Fisher syndrome and related disorders. J Neurol Neurosurg Psychiatry. 2012;83(9):941-948.

17. Funakoshi K, Kuwabara S, Odaka M, Hirata K, Yuki N. Clinical predictors of mechanical ventilation in Fisher/GuillainBarré overlap syndrome. J Neurol Neurosurg Psychiatry. 2009;80(1):60-64.

18. Shahrizaila N, Goh KJ, Kokubun N, Abdullah S, Yuki N. Serial nerve
Available online at: www.jccm.ro

conduction studies provide insight into the pathophysiology of Guillain-Barré and Fisher syndromes. J Neurol Sci. 2011;309(12):26-30.

19. Kim JE, Heo JH, Kim HO, et al. Neurological Complications during Treatment of Middle East Respiratory Syndrome. J Clin Neurol. 2017;13(3):227-233.

20. Li YC, Bai WZ, Hashikawa T. The neuroinvasive potential of SARSCoV2 may play a role in the respiratory failure of COVID-19 patients. J Med Virol. 2020;92(6):552-555. 